NISTIR 8260

\title{
Compression Guidance for 1000 ppi Palm and Hand Friction Ridge Imagery
}

John Libert

John Grantham

Craig Watson

This publication is available free of charge from: https://doi.org/10.6028/NIST.IR.8260

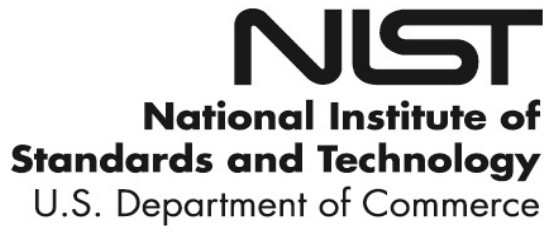




\title{
Compression Guidance for 1000 ppi Palm and Hand Friction Ridge Imagery
}

\author{
John Libert \\ Craig Watson \\ Information Access Division \\ Information Technology Laboratory
}

John Grantham Systems Plus, Inc.

Rockville, MD

This publication is available free of charge from: https://doi.org/10.6028/NIST.IR.8260

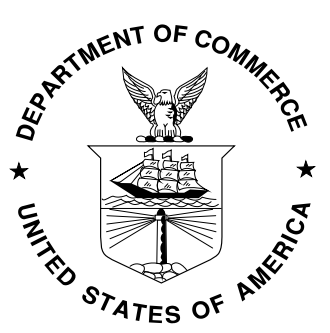

U.S. Department of Commerce Wilbur L. Ross, Jr., Secretary

National Institute of Standards and Technology Walter Copan, NIST Director and Undersecretary of Commerce for Standards and Technology 
Certain commercial entities, equipment, or materials may be identified in this document in order to describe an experimental procedure or concept adequately. Such identification is not intended to imply recommendation or endorsement by the National Institute of Standards and Technology, nor is it intended to imply that the entities, materials, or equipment are necessarily the best available for the purpose.

National Institute of Standards and Technology Interagency or Internal Report 8260 Natl. Inst. Stand. Technol. Interag. Intern. Rep. 8260, 24 pages (July 2019)

This publication is available free of charge from: https://doi.org/10.6028/NIST.IR.8260 


\begin{tabular}{|l|l|}
\hline Date & Activity \\
\hline & \\
\hline & \\
\hline & \\
\hline
\end{tabular}

\section{Disclaimer}

Certain commercial entities, equipment, or materials may be identified in this document to adequately describe an experimental procedure or concept.

Such identification is not intended to imply recommendation or endorsement by the National Institute of Standards and Technology, nor is it intended to imply that the entities, materials, or equipment are necessarily the best available for the purpose. 
In a previous special publication [NIST1], NIST provided guidance for the compression of 1000 ppi fingerprint images using Part I of the JPEG 2000 standard. The present document extends the Guidance to the compression of 1000 ppi palm and whole-hand images. The NIST Spectral Image Validation Verification (SIVV) metric is used to determine JPEG 2000 compression ratios at which palm and hand images exhibit spectral fidelity to non-compressed source images comparable to that exhibited by fingerprint images compressed at 10:1. The investigators discuss the effect of untextured regions (i.e. large areas of low-texture or "white space") in enabling compression ratios as high as 12:1 for palms and 22:1 for whole hand images as indicated by the spectral analysis. However, they note several factors that advise against deviating from the compression ratio recommended in the Guidance. Hence, NIST recommends that a single compression ratio, 10:1, be applied to all friction ridge impression types to ensure interoperability, in general, and support of latent matching, in particular.

KEYWORDS

palm print; hand print; JPEG 2000; friction ridge skin; Spectral Image Validation Verification metric, SIVV 


\section{TABLE OF CONTENTS}

1 Background ...................................................................................................................

2 Purpose ..................................................................................................................

3 Image Geometry and File Size..................................................................................4

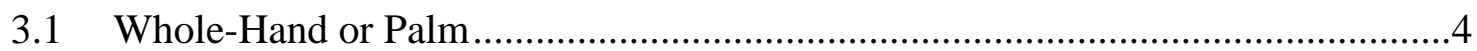

3.2 Single Rolled or Flat, Card Scan or Live Capture..................................................4

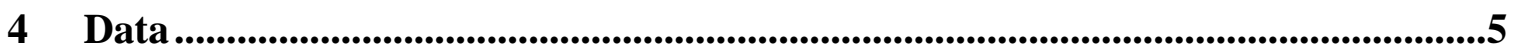

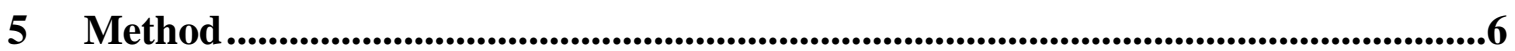

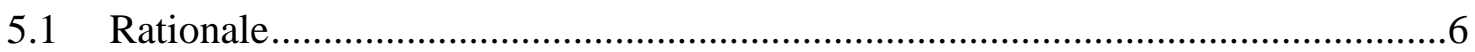

5.2 Frequency Spectrum Comparison ...................................................................6

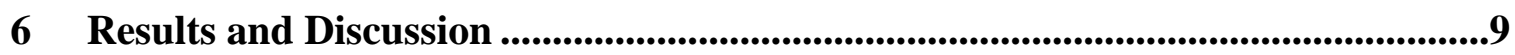

7 Compression Guidance ...............................................................................................11

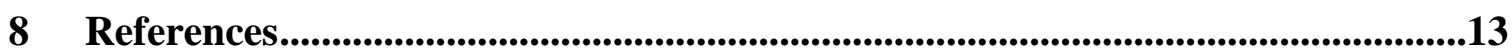

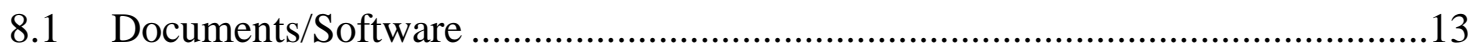

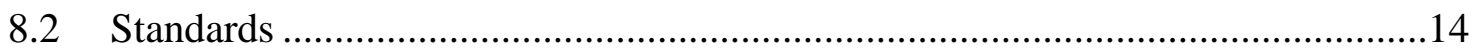




\section{LIST OF TABLES}

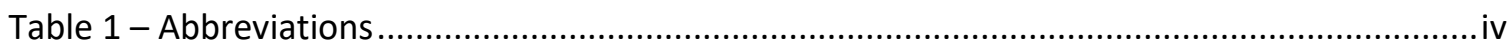

\section{LIST OF FIGURES}

Figure $1 \mathrm{SIVV}$ spectra, $\mathbf{s}_{\mathbf{1}}$ and $\mathbf{s}_{\mathbf{2}}$, of the two (synthetic) fingerprint impressions [CAPPELLI], img1 and img2, shown in the figure. Peak location corresponds to spatial frequency of ridge pattern. img2 results from a small degree of low-pass filtering applied to img1 reducing power in the high frequencies.

Figure 2 Mean response of the SIVV RMSE to the fingerprint baseline and to cropped palm and whole hand images.

Figure 3 Mixed Compression Ratio Cases (from [NIST1]) 
TERMS AND DEFINITIONS

Table 1 - Abbreviations

\begin{tabular}{|l|l|}
\hline ANSI & American National Standards Institute \\
\hline CJIS & Criminal Justice Information Services Division \\
\hline CODEC & Encoder and Decoder \\
\hline FBI & Federal Bureau of Investigation \\
\hline FTIR & $\begin{array}{l}\text { Frustrated Total Internal Reflection - optical principal employed by some fingerprint } \\
\text { acquisition devices }\end{array}$ \\
\hline IAI & International Association for Identification \\
\hline ISO & International Organization for Standardization \\
\hline ITL & Information Technology Laboratory \\
\hline NBIS & NIST Biometric Image Software \\
\hline NIST & National Institute of Standards and Technology \\
\hline PGM & Portable Graymap (image) Format \\
\hline ppi & Pixels per inch (the customary unit of sampling for digital fingerprints) \\
\hline ppmm & Pixels per millimeter \\
\hline SIVV & Spectral Image Validation/Verification Metric \\
\hline
\end{tabular}




\section{Executive Summary}

The criminal justice community has traditionally exchanged and stored fingerprint imagery data at 500 pixels per inch ${ }^{1}$ (ppi) or 19.7 pixels per millimeter (ppmm). The Wavelet Scalar Quantization (WSQ) fingerprint image compression algorithm is currently the standard for the lossy compression of 500 ppi fingerprint imagery. The WSQ Gray-Scale Fingerprint Image Compression Specification [WSQ] provides guidance based on an International Association for Identification (IAI) study [FITZPATRICK] to establish the acceptable amount of fidelity loss due to lossy compression in order for a WSQ encoder and decoder to meet FBI specifications for certification. These certifications are designed to ensure adherence to the WSQ specification and thereby to ensure fidelity and admissibility as forensic evidence of images that have been processed by such encoders and decoders.

Modern biometric systems are now trending towards the capture, transfer and storage of fingerprint images at $1000 \mathrm{ppi}$ or $39.4 \mathrm{ppmm}$ which offers many benefits, notably greater fidelity and better representation of Level 3 features ${ }^{2}$. The ANSI/NIST ITL and ISO 19794 standards require compression of 1000 ppi fingerprint imagery using the JPEG2000 algorithm rather than WSQ. Also, as systems transition to $1000 \mathrm{ppi}$, some systems will unavoidably contain an overlap between 500 ppi and 1000 ppi operational pathways. In addition to the adoption of JPEG2000, the overlap of 500 and 1000 ppi operational data will also require an interoperability bridge between traditional 500 ppi and modern 1000 ppi data.

NIST Special Publication 500-289 [NISTSP500-289] provides general guidance for the compression of 1000 ppi friction ridge imagery as well as an interoperability pathway between 500 ppi friction ridge imagery and new 1000 ppi friction ridge image data; the present document builds on that general guidance with more detailed guidance on the compression of 1000 ppi whole hand and palm images.

We apply a NIST-developed spectral analytic tool to set a spectral image fidelity baseline for fingerprint impressions determined by trained examiners to be of acceptable quality to support latent matching when compressed 10:1 using the NIST baseline JPEG 2000 CODEC. We, then, generalize this spectral fidelity baseline to both whole hand and cropped palm impressions to determine appropriate compression ratios. We find comparable fidelity levels for cropped palm at 12:1 and for whole hand images at 22:1.

We note that image degradation relative to target compression ratio is mitigated with the presence of impression areas lacking texture. The JPEG 2000 encoder can achieve a desired compression ratio with less quantization, i.e. information loss, with large areas of

\footnotetext{
${ }^{1}$ Resolution values for friction ridge imagery are specified in pixels per inch (ppi) throughout this document. This is based on widely used specification guidelines for such imagery and is accepted as common nomenclature within the industry.

2 The commonly accepted nomenclature defines Level 1 fingerprint details as the overall friction ridge pattern and flow, Level 2 detail as classic Galton features [GALTON] like minutiae points, and Level 3 as pores, creases, line shapes, incipient ridges and other non Level 1 or 2 features [JAIN].
} 
uniform gray level. Thus, whole hand images containing substantial untextured background may be compressed at 22:1 to achieve the spectral degradation comparable to the more tightly cropped fingerprint compressed at 10:1. However, as we note in NIST SP 500-289, selection from among multiple compression ratios would be difficult to handle reliably in operational settings; the extent of untextured area could be expected to vary over acquisition devices, increasing the likelihood that too much loss might be imposed on a tightly cropped hand impression; and machine matching tends to perform optimally when images under comparison have been subjected to similar compression levels. Accordingly, NIST recommends that palm and hand images, and other friction ridge impressions be compressed in accordance with the profile specified in NIST SP500-289, including use of the 10:1 compression ratio. The guidance in [NIST1] should also be followed with respect to lossless JPEG 2000 compression as well as approach to downsampling 1000 ppi images to $500 \mathrm{ppi}$. 


\section{Background}

The criminal justice community has traditionally captured, processed, stored and exchanged friction ridge imagery data at 500 pixels per inch ${ }^{3}$ (ppi), or 19.7 pixels per millimeter (ppmm), in the course of its operation. Modern biometric systems are trending towards operation on fingerprint images at $1000 \mathrm{ppi}$ or $39.4 \mathrm{ppmm}$. This transition to 1000 ppi friction ridge imagery offers many benefits, notably greater fidelity to the original sample and better representation of Level 3 features ${ }^{4}$. Both of these benefits may increase the probability of establishing a match/non-match decision by expert examiners and automated fingerprint matchers.

The JPEG2000 compression standard offers much flexibility in the types of images it can operate on as well as the way images can be compressed and encoded. While this flexibility can offer many operational benefits, the JPEG 2000 standard allows for many variations that present compatibility issues across the various stakeholders hindering interoperability among those stakeholders. Because of this, a need exists for a normative guidance that establishes a set procedure for the compression of images by all stakeholders. Adherence to this normative guidance by stakeholders provides assurances for compatibility between those stakeholders. NIST Special Publication 500-289 [NIST1] provides this normative guidance for compression of grayscale friction ridge imagery at 1000 ppi.

While the guidance in NIST Special Publication 500-289 [NIST1] provides a solid baseline for the compression of friction ridge imagery of all types, there exists a wide variety of impression types, some of which may allow for, or even benefit from, additional compression beyond the level recommended in [NIST1]. Whole hand and palm images are in the range of one, or even two, order(s) of magnitude (approximately 10 to 150 times) larger, in terms of both image geometry and file size, than single-finger impression types (see section 3). These larger images not only contain a significantly larger amount of meaningful friction-ridge information, but also include additional superfluous background information, such as the mildly textured fingerprint card on which the impression was originally taken or "white space" in the case of a live-scan fingerprint capture device. This additional background or "white space" could allow for more compression than is applied to single-finger impression types which typically contain much less background information.

Even a small amount of additional compression can have a significant impact on the resulting file size of a compressed whole-hand or palm image. For example, compressing an image at 10:1 results in a reduction in filesize of approximately $90 \%$ (as 10:1

\footnotetext{
${ }^{3}$ Resolution values for friction ridge imagery are specified in pixels per inch (ppi) throughout this document. This is based on widely used specification guidelines for such imagery and is accepted as common nomenclature within the industry. SI units for these will be presented only once in the body of this document.

${ }^{4}$ The commonly accepted nomenclature defines Level 1 fingerprint details as the overall friction ridge pattern and flow, Level 2 detail as classic Galton features [GALTON] like minutiae points, and Level 3 as pores, creases, line shapes, incipient ridges and other non-Level 1 or 2 features [JAIN].
} 
compression means that the result is 10 times smaller than the original). Increasing the ratio to 20:1 results in a reduction of $95 \%$, or an additional reduction of $5 \%$ of the original file size. This may not seem significant, but with some fingerprint imagery databases containing potentially several million whole-hand images, the total savings in terms of storage space - and bandwidth in the case of transmitting fingerprint records could be quite significant. 


\section{Purpose}

The purpose of this publication is to provide guidance for both the lossy and lossless compression of 1000 ppi grayscale whole-hand and palm friction-ridge images. This specialized guidance is derived from and extends the generalized guidance for the compression of friction ridge imagery provided in [NIST1]. 


\section{Image Geometry and File Size}

This section provides the basis for the various image geometries to which this guidance applies.

\subsection{Whole-Hand or Palm}

The ANSI/NIST [AN2k11] and EBTS [CJIS] standards define the following multi-finger impression geometries:

- $\quad$ EBTS 10.0.9 specifies image dimensions 5.5" x 8.0"5, or $139.7 \mathrm{~mm}$ x $203.2 \mathrm{~mm}$, (44 million pixels at $1000 \mathrm{ppi}$ ) for a full palm impression.

- $\quad$ EBTS 10.0.9 indicates 5.5" x 5.5", or $139.7 \mathrm{~mm}$ x 139.7, (30.25 million pixels at 1000 ppi) for a half palm impression.

- ANSI/NIST-ITL 1-2011 specifies image dimensions of 5.5" x 8.5", or $139.7 \mathrm{~mm}$ x $215.9 \mathrm{~mm}$, (46.75 million pixels at $1000 \mathrm{ppi}$ ) for a full palm impression.

- ANSI/NIST-ITL 1-2011 specifies image dimensions of 5.5" x 5.5" (30.25 million pixels at $1000 \mathrm{ppi}$ ) for half palm impression.

\subsection{Single Rolled or Flat, Card Scan or Live Capture}

The ANSI/NIST [AN2k11] and EBTS [CJIS] standards define the following single impression geometries:

- A rolled impression at 1.6” x 1.5”, or $40.64 \mathrm{~mm}$ x $38.1 \mathrm{~mm}$, in size or approximately 2.4 million pixels at 1000 ppi (EBTS 10.0.9 [CJIS]).

- A flat (plain) impression up to 1" x 2", or $25.4 \mathrm{~mm}$ x $50.8 \mathrm{~mm}$, in size or approximately 2.0 million pixels at 1000 ppi (EBTS 10.0.9 [CJIS]).

- ANSI/NIST-ITL 1-2011 [AN2k11] specifies that rolled impression images can be up to 1.6 ” x 1.5 " in size (2.4 million pixels at $1000 \mathrm{ppi}$ ).

- ANSI/NIST-ITL 1-2011 specifies that flat (plain) impression images can be up to 1.0 ” x 3.0", or $25.4 \mathrm{~mm}$ x $76.2 \mathrm{~mm}$, in size (3.0 million pixels at $1000 \mathrm{ppi}$ ).

- The Personal Identity Verification [PIV] specification calls for single finger capture images to be at least $12.8 \mathrm{~mm}$ by $16.5 \mathrm{~mm}$ high $+-0.02 \%$ (approximately 0.504 " x 0.650 " or 302400 pixels at $1000 \mathrm{ppi}$ ).

Based on the above, the normal supported operational range for multi-finger slap or palm impressions (for both Card Scan and Live Capture) is expected to be from 30250000 pixels to 46750000 pixels at 1000 ppi, while the range for Single Rolled or Flat fingerprint imagery geometry (for both Card Scan and Live Capture) is expected to be from 302400 pixels to 3000000 pixels at $1000 \mathrm{ppi}$. This difference of at least one order of magnitude represents a very significant difference in the nature of these two categories of impression types [NIST1].

\footnotetext{
${ }^{5}$ Geometry values for friction ridge imagery are specified in inches throughout this document. This is based on widely used specification guidelines for such imagery and is accepted as common nomenclature within the industry. SI units for these will be presented only once in the body of this document.
} 


\section{Data}

For this study, an image dataset was prepared by scanning inked impressions of both right and left whole-hands of 100 subjects. The 200 cards were scanned using an Epson V850 digital flatbed scanner with customized software written for scanning these cards ${ }^{6}$ at 1000 ppi. The whole-hand impressions were then extracted from the initial full-card scan images using the bounding boxes defined on the fingerprint cards and rotated into an upright position, with fingertips positioned at the top of the image.

Palm-only images were extracted from the whole-hand images using manual segmentation, i.e. cropping the palms from the whole hand images. The scanning and segmentation operations resulted in a set of 200 whole-hand images and 200 palm-only images.

\footnotetext{
${ }^{6}$ NISTScan is a Windows application written in C\#/.NET which uses the TWAIN protocol to acquire images from scanning devices (such as, but not limited to, the Epson V850).
} 


\section{Method}

\subsection{Rationale}

The guidance for the application of JPEG 2000 to compression of 1000 ppi fingerprints [NIST1] had benefit of inspection of multiple trained fingerprint examiners. The outcome of several studies [NIST1, NIST3, NIST4, NIST6, NIST7, NIST8, NIST9] in support of this guidance was the recommendation that 10:1 compression would best ensure preservation of detail to support forensic fingerprint examination, including latent matching. As will be further discussed later, some impression types having large areas of white space (non-textured regions) could tolerate higher compression rates, but 10:1 compression was recommended as the most conservative and likely to avoid mistakes in selection of an inappropriate compression rate.

As we do not have benefit of examiner assessment, we can select a suitable compression rate for palm images by using computational measurements to determine levels of compression comparable to those applied to fingerprints. Thus, we apply metrics to fingerprint images used in the examiner studies to determine compression levels for palm images at similar values of metrics.

The full set of 400 original images described above was compressed at 2:1, 5:1, 7:1, 10:1, 12:1, 15:1, 17:1, 20:1, 22:1, 26:1, 30:1, 34:1, and 38:1, using the NIST JPEG2000 Reference Encoder, CJP2K [NIST5]. CJP2K implements the parameters and settings detailed in NIST Special Publication 500-289 [NIST1].

The compressed images were then measured using the metric described in the following sections.

\subsection{Frequency Spectrum Comparison}

The NIST Spectral Image Validation/Verification (SIVV) Metric [NIST2] was developed initially as a method to screen fingerprint databases for non-fingerprint images, segmentation errors, or mislabeled sample rates. As a 1-dimensional representation of a 2-dimensional Fourier spectrum, the SIVV metric applied to a fingerprint image exhibits a major peak corresponding to the average frequency of the ridge spacing. Frequency and amplitude of this peak as well as other features of the 1D signal can be used to differentiate fingerprints from other images contaminating a fingerprint database.

The SIVV also provides a comparatively straightforward method by which to assess the fidelity of a modified image to its unprocessed source. Pairwise display of the SIVV signals of two images enables summary visualization of the effects of a process on the structure of a source image. For example, as shown in Figure 1, comparison of SIVV signals of two synthetic fingerprint impressions shows the difference in spectral power over various frequencies of a source image (img1) and that resulting (img2) from some low-pass filtering applied to the source image. 
The SIVV signals denoted as $\mathbf{s}_{\mathbf{1}}$ and $\mathbf{s} \mathbf{2}$ are respectively vectors of SIVV signal values for images under comparison. The number of frequency samples, $f$, in units of cycles per pixel, correspond to one-half the length (number of pixels) of the smaller dimension of an image. Frequency along this dimension is scaled to the interval $[0,0.5]$ cycles/pixel because the smallest possible cycle is manifested by two pixels (and $1 / 2=0.5$ ). Note that the power value at $f=0$ is the "direct current" (DC) term, corresponding to the average intensity of the image and is used to normalize the power spectrum.

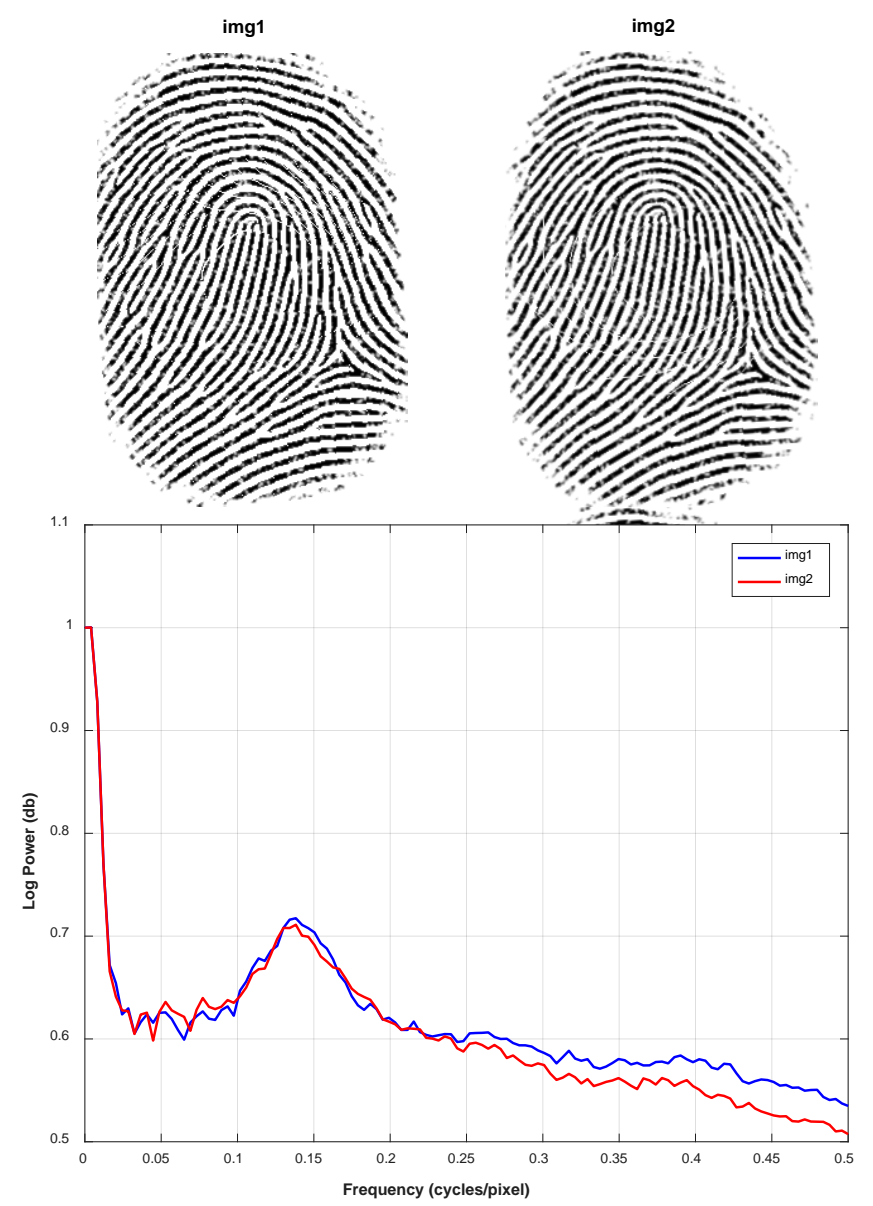

Figure 1 SIVV spectra, $\mathbf{s} 1$ and $\mathbf{s} 2$, of the two (synthetic) fingerprint impressions [CAPPELLI], img1 and img2, shown in the figure. Peak location corresponds to spatial frequency of ridge pattern. img2 results from a small degree of low-pass filtering applied to img1 reducing power in the high frequencies.

\subsubsection{RMS Error of SIVV Signals}

Either differences or ratios of SIVV signals can provide quantitative measures for the effects of compression or other processes applied to images. For the present study, we examine image differences between pairs of images, $\mathbf{I}^{\prime} \mathbf{A}$ and $\mathbf{I}_{\mathbf{B}} \mathbf{B}$, with respect to the Root Mean Squared Error (RMSE) between their two SIVV signals, s1 and s2, over the entire frequency range 0 - 0.5 cycles/pixel. 


$$
\operatorname{RMSE}(\mathbf{s} 1, \mathbf{s} 2)=\sqrt{\frac{\sum_{i=1}^{n}\left(\mathbf{s} 1_{i}-\mathbf{s} 2_{i}\right)^{2}}{n}}
$$

where $n=|\mathbf{s} 1|=|\mathbf{s} 2|$ (i.e., the lengths of the signal vectors).

The RMSE metric defined above can provide a measure of the overall difference between the SIVV spectra of images subjected to different processes or, as in the present study, friction ridge impressions subjected to varying levels of JPEG 2000 compression. In addition to global effects, the RMSE may be evaluated over smaller frequency intervals enabling the comparison of effects over frequency bands that may have particular relevance to fingerprint image quality or matching. The SIVV RMSE also quantifies and isolates changes confined to bands that specifically impact either the machine matcher or expert examiners. In the present case, however, as the images are identical except for relative loss due to compression, the RMSE over the entire frequency range is used, even though most of the differences between paired signals occur at middle-to-high frequencies. 


\section{Results and Discussion}

Figure 2 plots the mean RMSE for 200 SIVV signal comparisons between compressed and non-compressed source images at each of the thirteen target compression ratios listed above. Separate plots are shown for fingerprints (as used in support of fingerprint compression guidance in [NIST1]), cropped palms, and whole hand images scanned from cards. The dot-dashed (reference) line on the graph marks the RMSE at 10:1 compression for fingerprints; 10:1 was found to be optimal per the assessment of trained examiners reported in [NIST1] and [NIST3]. The intersection of this reference line with the plots for cropped palm and whole hand mark comparable mean SIVV RMSE for each of these impression types. This marks equivalent levels of degradation to those found acceptable for fingerprints.

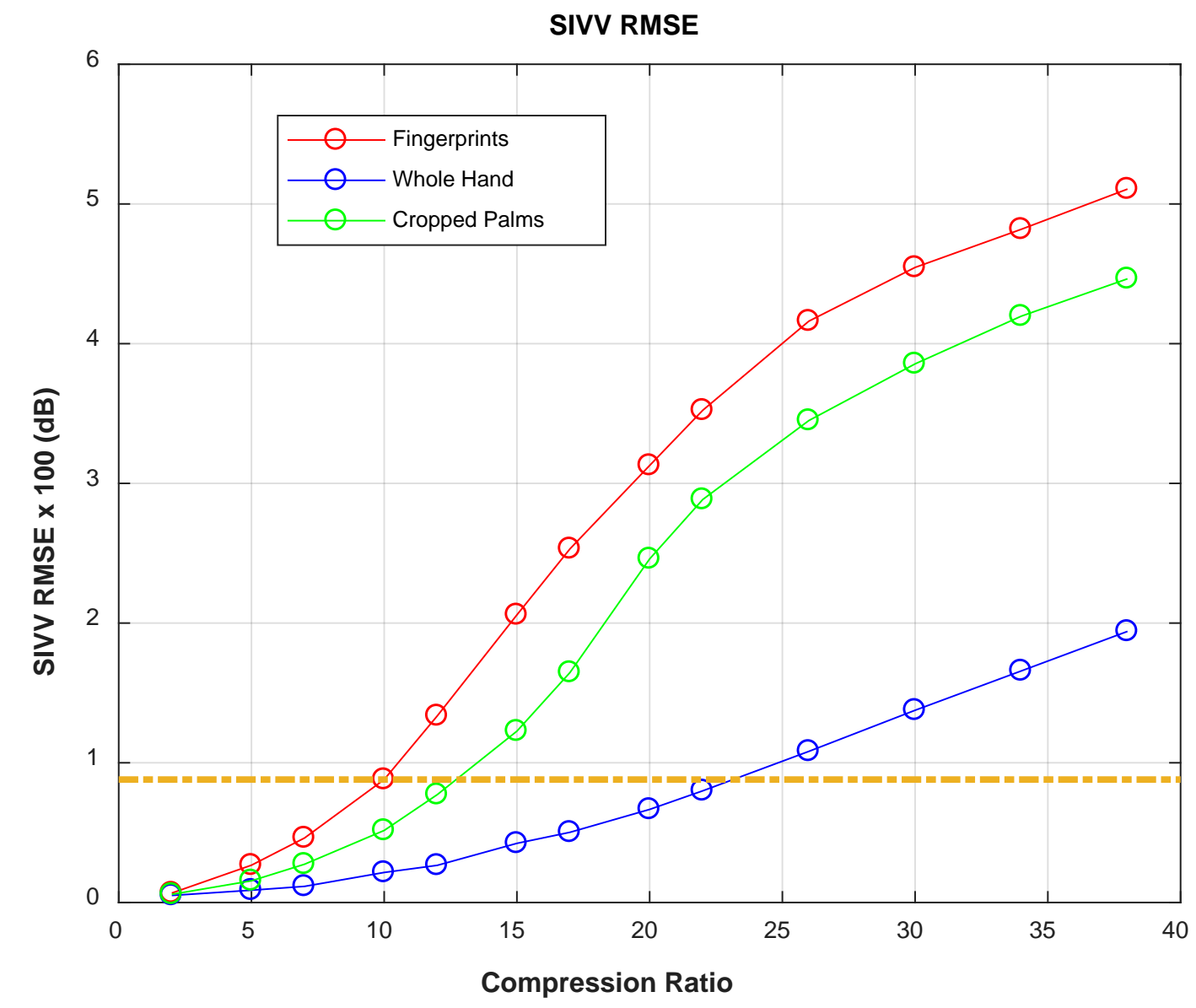

Figure 2 Mean response of the SIVV RMSE to the fingerprint baseline and to cropped palm and whole hand images.

We can convert this into suggested JPEG 2000 compression ratios by dropping a vertical to the abscissa of the graph at each of the intersection points to determine the comparable compression ratios for palm/hand impressions. We note that while the graph connects markers with lines, there are really no actual measurements other than at marker points. 
We use the marker nearest the intersection of the plot with the reference line to indicate the appropriate compression ratio for each impression type. Thus, for cropped palm, the data suggest 12:1 as the compression rate target. For whole hand, an acceptable compression ratio would appear to be 22:1.

However, we faced similar cases in development of the compression guidance [NIST1]. In [NIST3], we noted that while card-scanned inked fingerprints required a compression ratio of 10:1, livescan (FTIR) acquisitions could tolerate compression at 15:1. We noted in [NIST1] that research results indicated that machine matching tends to perform best when images are compressed at the same rate (see section 7). We further reasoned that specification of different compression rates for various friction ridge impression types might be prone to error in operational settings where the incorrect compression ratio might accidently be applied to an impression type or mode of acquisition. We concluded that the most conservative approach to ensure image quality in both machine matching as well as latent matching applications would be to use a single compression rate specification for all friction ridge impressions.

We find a similar situation for palm/hand prints. The spatial frequency data show that cropped palms could be compressed at 12:1 and whole hand images at 22:1. Compression ratios higher than the 10:1 specified in [NIST1] appear acceptable because of the greater areas of low texture or "white space" in these impressions. As explained in [NIST4], unlike the WSQ encoder, the JPEG 2000 encoder adjusts the information loss to meet the compression rate specification. In this case, white space enables less aggressive quantization of the friction ridge region of the impression. Given a rate specification, the WSQ encoder allows the actual compression ratio to vary to achieve a consistent image quality.

We sampled the whole hand prints by segmenting the entire area contained in the bounding box inscribed on FD-884 card. No effort was made to trim extraneous white space, nor would it be possible to remove the white space between the fingers.

Undoubtedly, this enabled compression of whole hand images at 22:1. Yet, were a tighter cropping applied to these images, the indicated compression ratio would be closer to 15:1 or even 12:1. Accordingly, while tempting, compression of hand images at greater than 15:1 (or even 12:1) would be ill advised. 


\section{Compression Guidance}

NIST Special Publication 500-289 [NIST1] recommends lossless compression of latent imagery using the JPEG2000 reversible filter, and lossy 10:1 compression with JPEG2000 for other impression types.

While an optimal solution for compression rate would be to select 10:1 for card scan imagery and 15:1 for live scan imagery, experimental results show mixed compression rates can negatively impact the matcher, for example, where a 10:1 card scan image is compared to a 15:1 live scan image in the gallery. In this experiment a set of 2448 cardscan fingerprint images were compressed at 2:1, 5:1, 7:1, 10:1, 12:1, 15:1 and 17:1. The fingerprint images were matched against themselves (same impression, same image) at several different combinations of compression ratios as shown in Figure 3. The images of different identities were also matched against each other as 496944 non-mate cases using the same compression ratio combinations. For the specific mixed compression scenario of 10:1-to-15:1, the median score obtained from matching mated images was 224 while the same images in the 10:1-to-10:1 scenario yielded a median match score of 499 . These results were determined to be statistically significant using Wilcoxon signed rank test $(\mathrm{P}<0.0001)$.

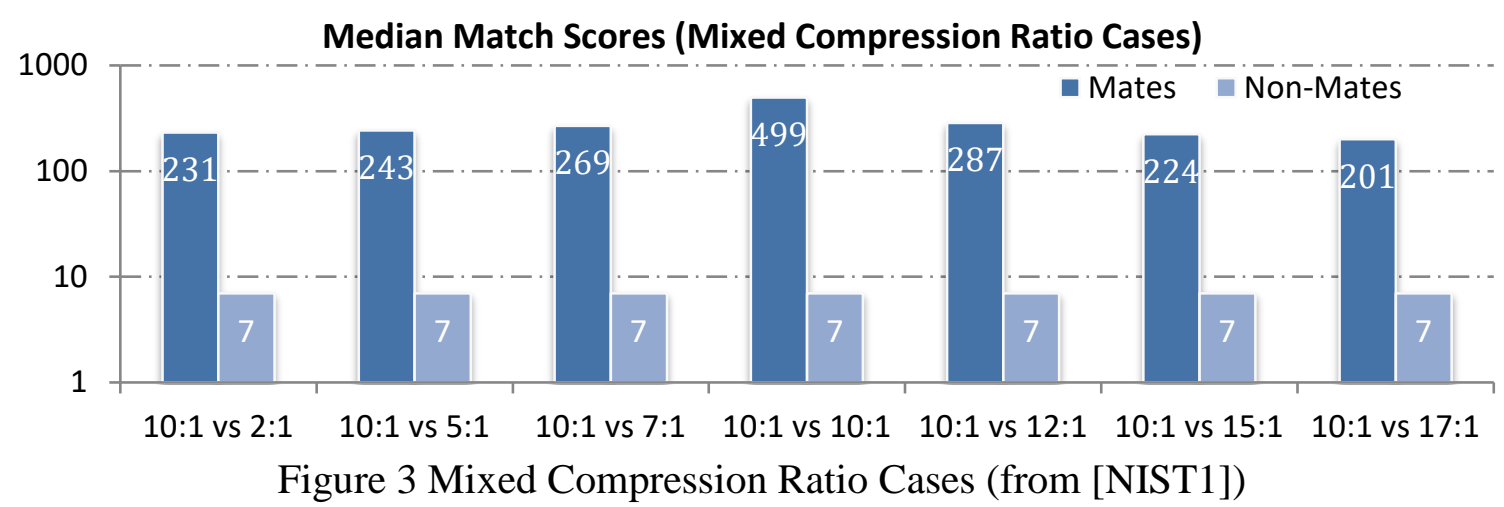

Exploratory testing of automated matcher behavior using lossy compressed images for [NIST1] showed a tendency by the automated matcher to favor like-vs.-like cases in terms of compression ratio (see Figure 3 ) and there was a slight penalty where the compression ratio of the probe differed from that of the gallery.

Higher rates of compression were found to be acceptable for some fingerprint impression types where the image includes considerable untextured space. In this regard, we note that JPEG 2000 applies less aggressive treatment to the friction ridge texture in the presence of untextured space. Livescan and slap impressions that have relatively untextured backgrounds allows the JPEG 2000 algorithm to achieve the target compression level with less degradation of the friction ridge area. Despite this, in [NIST1] we recommended that a single conservative compression level be used rather than different levels, selected for each impression type, to reduce the likelihood of users accidently selecting an inappropriate compression target for their images. Additionally, at the potentially suitable higher compression ratios found above, 12:1 for palm-only 
images and 22:1 (adjusted to 15:1 or 12:1 by the argument presented above) for wholehand images, the additional reduction in file size may not be significant enough to justify the issues presented by matching in a mixed-ratio environment and the risk of human error.

For example, as described above in section 3, a typical whole-hand image is 5.5” x 8.5" (5500 x 8500 pixels at $1000 \mathrm{ppi}$ ), which equates to 46750000 bytes of image data. When compressed at 10:1, the resulting file is 10 times smaller at approximately 4675000 bytes for a file size reduction of $90 \%$ over the original uncompressed image. When the compression ratio is increased to 12:1 the resulting file is 3895834 bytes for a reduction of $\approx 92 \%$ over the original uncompressed image. In this scenario, the higher compression ratio saves an additional 779166 bytes, or $\approx 2 \%$, in storage space over the lower compression ratio. In a database with one million such images, the overall additional savings in storage space for images compressed at the higher ratio would amount to approximately 709 gigabytes (709 000000000 bytes). At 22:1, the file size reduction for one image is $\approx 95 \%$ for an additional savings of 2550000 bytes (as compared to 10:1); for a set of one million images this results in an additional savings of approximately 2.3 terabytes (2 300000000000 bytes).

As a result of the aforementioned considerations, we follow a conservative approach and recommend that the 10:1 compression specification be applied to palm and hands as well as single-finger and slap-four impressions. 


\section{References}

\subsection{Documents/Software}

CAPPELLI Cappelli, R., Maio, D., and Maltoni, D. SFinGe: An Approach to Synthetic Fingerprint Generation, International Workshop on Biometric Technologies, 2004. (Demo. software available at http://biolab.csr.unibo.it/research.asp?organize=Activities\&select=\&selObj=12\&pathSubj=111\%7C\%7C12\&

FITZPATRICK Fitzpatrick, M. et al. 1994, "WSQ Compression / Decompression Algorithm Test Report”, IAI Annual Conference

GALTON Galton, F. (2005). Finger prints. Mineola, NY: Dover Publications. (Original work published 1892)

JAIN Jain, A., "Pores and Ridges: High-Resolution Fingerprint Matching Using Level 3 Features”, IEEE Transactions on Pattern Analysis and Machine Intelligence, Vol. 29, No. 1, January 2007.

NIST1 Orandi, S., Libert, J.M., Grantham, J.D., Ko., K., Wood, S. S., Byers, F., Bandini, B., and Harvey, S.G., Compression Guidance for 1000 ppi Friction Ridge Imagery, NIST Special Publication 500-289, National Institutes of Standards and Technology, Gaithersburg, MD., February 2014. http://nvlpubs.nist.gov/nistpubs/specialpublications/NIST.SP.500-289.pdf Retrieved 07/31/2017.

NIST2 Libert, J., Grantham, J., Orandi, S. A 1D Spectral Image Validation/Verification Metric for Fingerprints, NIST Interagency Report 7599, August, 2009 http://ws680.nist.gov/publication/get_pdf.cfm?pub_id=903078

NIST3 Orandi, S., Libert, J. M., Grantham, J. D., Ko, K., Wood, S.S., Wu, J., "Effects of JPEG2000 Image Compression on 1000 ppi Fingerprint Imagery”, NIST Interagency Report 7778, National Institutes of Standards and Technology, Gaithersburg, MD. http://www.nist.gov/customcf/get_pdf.cfm?pub_id=908204. Retrieved 09/01/2012.

NIST4 Libert, J, M., Orandi, S., and Grantham, J. D. Comparison of the WSQ and JPEG 2000 Image Compression Algorithms On 500 ppi Fingerprint Imagery. NIST Interagency Report 7781, National Institutes of Standards and Technology, Gaithersburg, MD., April 24, 2012.

http://ws680.nist.gov/publication/get_pdf.cfm?pub_id=910658 Retrieved 08/08/2017.

NIST5 "NIST Biometric Image Software". http://Fingerprint.nist.gov/NFIS/. Retrieved 2011-01-12.

NIST6 Orandi, S., Libert, J. M., Grantham, J. D., Ko, K., Wood, S. S., Wu, J. C., Petersen, L. M., Bandini, B., “An Exploration of the Operational Ramifications of Lossless Compression of 1000 ppi Fingerprint Imagery”, NIST Interagency Report 7779, National Institute of Standards and Technology, Gaithersburg, MD. http://www.nist.gov/customcf/get_pdf.cfm?pub_id=911122. Retrieved 03/29/2013.

NIST7 Orandi, S., Libert, J.M., Grantham, Petersen, L. P., "Effects of JPEG 2000 Lossy Image Compression on 1000 ppi Latent Fingerprint Imagery”, NIST Interagency Report 7780, National Institutes of Standards and Technology, Gaithersburg, MD. http://www.nist.gov/customcf/get_pdf.cfm?pub_id=914513. Retrieved 07/31/2013.

NIST8 Orandi, S., Libert, J.M., Grantham, J.D., Lepley, M., Bandini, B., Ko., K., Petersen, L. P., Wood, S. S., and Harvey, S.G., "Examination of Downsampling Strategies for Converting 1000 ppi Imagery to 500 ppi”, NIST Interagency Report 7839, National Institutes of Standards and Technology, Gaithersburg, MD. http://nvlpubs.nist.gov/nistpubs/ir/2013/NIST.IR.7839.pdf. Retrieved 03/11/2013.

NIST9 Libert, J. M., Orandi, S., Grantham, J.D., “Effects of Decomposition Levels and Quality Layers with JPEG 2000 Compression of 1000 ppi Fingerprint Images”, NIST Interagency Report 7939, National Institutes of Standards and Technology, Gaithersburg, MD. http://nvlpubs.nist.gov/nistpubs/ir/2013/NIST.IR.7939.pdf. Retrieved 11/18/2013. 


\subsection{Standards}

\begin{tabular}{|c|c|}
\hline 12k11 & $\begin{array}{l}\text { Special Publication (NIST SP) - 500-290e3: “American National Standard for Information Systems } \\
\text { - Data Format for the Interchange of Fingerprint, Facial \& Other Biometric Information, } \\
\text { ANSI/ NIST-ITL 1-2011 NIST Special Publication 500-290 Edition 3. August 22, } 2016 . \\
\text { http:// nvlpubs. nist.gov/ nistpubs/ SpecialPublications/ NIST.SP.500-290e3. pdf }\end{array}$ \\
\hline CJ IS & $\begin{array}{l}\text { FBI/CJ IS, "Electronic Biometric Transmission Specification (EBTS) Technical and Operational } \\
\text { Update (TOU) 10.0.9 Redlines", Federal Bureau of Information Criminal J ustice Information } \\
\text { Systems, Clarksbug, WV, May 22, 2018, } \\
\text { https:// www.fbibiospecs. cjis. gov/ Document/ Get?fileName=EBTS\%20TOU\%2010 } 09 \text { Final. pdf } \\
\text { Retrieved 05/28/2019 }\end{array}$ \\
\hline J PEG & $\begin{array}{l}\text { "T. } 81 \text { : Information technology - Digital compression and coding of continuous-tone still images - } \\
\text { Requirements and guidelines". http:// www. itu. int/ rec/ T-REC-T.81. Retrieved 2011-01-12. }\end{array}$ \\
\hline J PEG2K & $\begin{array}{l}\text { "ISO/IEC 15444-1:2004 - Information technology -- J PEG2000 image coding system: Core coding } \\
\text { system". } \\
\text { http:// www. iso.org/ iso/ iso catalogue/ catalogue ics/ catalogue detail_ics. htm?csnumber=27687. } \\
\text { Retrieved 2009-11-01. }\end{array}$ \\
\hline PIV & $\begin{array}{l}\text { “Personal Identity Verification (PIV) Image Quality Specifications for Single Finger Capture } \\
\text { Devices”, FBI/ CJ IS Biometric Specifications, } 10 \text { J uly 2006, } \\
\text { https:// www.fbibiospecs. cjis. gov/ Document/ Get?fileName=pivspec.pdf. Retrieved 04/ 18/2019 }\end{array}$ \\
\hline WSQ & $\begin{array}{l}\text { "WSQ Gray-Scale Fingerprint Image Compression Specification" Version 3.1. } \\
\text { https:// www. fbibiospecs. org/ docs/WSQ Gray-scale Specification Version } 31 \text { Final.pdf. } \\
\text { Retrieved 2010-01-11. }\end{array}$ \\
\hline
\end{tabular}

